\title{
APPROXIMATE INPUT-OUTPUT LINEARIZATION OF NONLINEAR SYSTEMS USING THE OBSERVABILITY NORMAL FORM
}

\author{
K. Guemghar, B. Srinivasan, D. Bonvin \\ Laboratoire d Automatique, Ecole Polytechnique Federale de Lausanne. CH-1015 Lausanne, Switzerland \\ kahina.guemghareepfl.ch, bala.srinivasandepfl.ch, dominique.bonvineepfl.ch
}

Keywords: Nonlinear systems, Feedback linearization, Nonminimum-phase systems, Vanishing perturbations.

\begin{abstract}
The problem of controlling nonlinear nonminimum-phase systems is considered, where standard input-output feedback linearization leads to unstable internal dynamics. This problem is handled here by using the observability normal form in conjunction with input-output linearization. The system is feedback linearized upon neglecting a part of the system dynamics, with the neglected part being considered as a perturbation. A linear controller is designed to accommodate the perturbation resulting from the approximation. Stability analysis is provided based on the vanishing perturbation theory.
\end{abstract}

\section{Introduction}

The control of nonlinear systems is a challenging field for research and applications, especially since there exists no generic methods for controller synthesis $[10,18]$. One major research direction is the use of control Lyapunov functions [10]. However, there are no systematic ways of constructing a control Lyapunov function except for systems that are passive [15] or for triangular systems where back-stepping can be applied [11].

Another direction of investigation has dealt with feedback linearization. Therein, the nonlinearities of the system are first compensated using nonlinear state feedback and a nonlinear state transformation. Then, a linear controller is designed to control the linearized system [8]. The main limitation is that there are many systems for which the entire system nonlinearity cannot be compensated. Hence, various ideas related to linearization have been explored in the literature, for systems that are not full-state feedback linearizable [5].

- Approximate full-state linearization: (i) In [2], nonlinear state feedback and a coordinate transformation are used to make the system as close as possible to a linear one. (ii) Also, in [16, 19], pseudo-linearization is investigated, which consists of using nonlinear state feedback and a coordinate change such that the Taylor series linearization of the transformed system is independent of the operating point around which it is linearized. Necessary and sufficient conditions for pseudo-linearization have been computed.

- Input-output linearization: The idea of linearizing only part of the dynamics, i.e. the dynamics between the input and the output, has been widely used [8]. In such a scheme, referred to as input-output linearization, the nonlinearities are pushed to the so-called internal dynamics. In the context of input-output linearization, the main consideration is that the internal dynamics can be unstable. If the input-output behavior is minimum phase, the internal dynamics are stable, whereas they are unstable otherwise. So, instead of using the predefined system output, efforts have been concentrated on searching for new outputs for which the internal dynamics are stable $[4,13]$. The main difficulty with the above approach is that the performance as viewed from the predefined output could be unsatisfactory. In this paper, the outputs are predefined, and no new minimum-phase outputs are searched for.

- Input-output linearization with stabilization of internal dynamics: (i) In the case where minimum-phase outputs cannot be found, one possibility is to stabilize the internal dynamics. Though this problem is addressed in [7], no systematic approach to design the controller that stabilizes the internal dynamics is provided. (ii) In [3], a trajectory planning approach is used to provide a stable inversion of the system. (iii) On the other hand, a cascade control scheme has been considered that combines inputoutput feedback linearization and predictive control [6]. However, the scheme can only be applied to systems with slow inverse dynamics due to the time limitation imposed by the predictive control approach.

- Input-output linearization with approximate internal dynamics: (i) In [1], Taylor series linearization of the internal dynamics is used. Also, the internal dynamics are factored into decoupled unstable and stable parts. With this approach, the stability is only local. (ii) In [9, 17], the outputs are differentiated as many times as the order of the system instead of stopping at the relative degree as would be done with standard input-output linearization. With this, the input derivatives that appear in the control law are set to zero in the computation of the feedback input. Though global stability can be established, the main disadvantage of this method is that it requires the open-loop system to be stable. (iii) In [12], the pseudo- 
linearization method is generalized to the input-output linearization problem, where a sufficient and necessary condition for existence of a state feedback and a state coordinate change is given, such that the transformed system is input-output pseudo-linearized. With this approach, the stability is local.

This paper falls under the last category of methods, i.e. approximate input-output linearization. The idea used here is similar to $[9,17]$, though a more standard representation, i.e. the observability normal form will be exploited. Instead of setting the input derivatives to zero, part of the dynamics are neglected so as to make the approximate system feedback linearizable. The neglected part is then considered as a perturbation, and stability is analyzed using the vanishing perturbation theory. The idea is to design the linear controller so as to account for the nonlinearities that have not been compensated but simply neglected. This way, for the stability of the closed-loop system, there is no necessity that the open-loop system be stable.

Note that, once an appropriate part of the dynamics is neglected, the system becomes full-state feedback linearizable, with the derivatives of the predefined outputs providing the desired nonlinear state transformation. So, this method could also be classified as approximate full-state linearization, though it is preferable to keep it under input-output linearization due to the important role played by the predefined outputs. In other words, the predefined outputs, which were nonminimum phase for the original system, become minimum phase with no internal dynamics for the approximate system.

The paper is organized as follows: Section 2 introduces the basic concepts and notations in the fields of input-output feedback linearization and the theory of vanishing perturbations. Section 3 develops the proposed approximate linearization approach and the stability analysis of the scheme. Section 4 uses an example to illustrate the proposed method, and Section 5 concludes the paper.

\section{Preliminaries}

\subsection{Input-output Feedback Linearization}

Consider the single-input single-output nonlinear affine-ininput system represented by:

$$
\begin{aligned}
& \dot{x}=f(x)+g(x) u, \quad x(0)=x_{0} \\
& y=h(x)
\end{aligned}
$$

where $x \in R^{n}$ are the states, $u \in R$ the input, $y \in R$ the output, $x_{0}$ the initial conditions, and $f$ and $g$ the functions describing the system dynamics. Assume that $x=0, u=0$ is an equilibrium point with $f(0)=0$. $h$ is the output function with $h(0)=0$.

The relative degree, $r$, is the number of times the output has to be differentiated with respect to time before the input appears. System (1)-(2) can be input-output linearized into ByrnesIsidori normal form using the following steps [8]:
1. Apply a state feedback law that compensates the nonlinearities in the input-output behavior:

$$
u=\frac{v-L_{f}^{r} h(x)}{L_{g} L_{f}^{r-1} h(x)}
$$

where $L_{f} N(x)=\frac{\partial N}{\partial x} f(x)$ is the Lie derivative of $N$. Since $r$ is the relative degree, and $L_{g} L_{f}^{i} h(x)=0, \forall i<$ $r-1, \forall x, L_{g} L_{f}^{r-1} h(x) \neq 0$.

2. Use the nonlinear transformation $z=T(x), z=$ $\left[y \dot{y} \cdots y^{(r-1)} \eta^{T}\right]^{T}$, with $\eta \in R^{n-r}$, to express System (1)-(2) as:

$$
y^{(r)}=v, \quad \dot{\eta}=\mathcal{Q}\left(\eta, y, \dot{y}, \cdots, y^{(r-1)}, v\right)
$$

\subsection{Theory of Vanishing Perturbations}

Consider the dynamic system

$$
\dot{x}=\Psi(x)+\Delta(x)
$$

where $\Psi$ is the nominal dynamics and $\Delta$ the perturbed dynamics, both Lipschitz in $x$. Also let $\Psi(0)=0$.

The vanishing perturbation theory is based on the assumption that $\Delta(0)=0$. Since $\Delta(x)$ is Lipschitz,

$$
\|\Delta(x)\| \leq \delta\|x\|
$$

The main result is that, if the nominal system is exponentially stable and $\delta$ is smaller than a predetermined limit, then the perturbed system is also exponentially stable.

Theorem 1 [10] Let $x=0$ be an exponentially stable equilibrium point of the nominal system $\dot{x}=\Psi(x)$. Let $V(x)$ be a Lyapunov function for the nominal system that satisfies

$$
\begin{gathered}
\frac{\partial V}{\partial x} \Psi(t, x) \leq-c_{1}\|x\|^{2} \\
\left\|\frac{\partial V}{\partial x}\right\| \leq c_{2}\|x\|
\end{gathered}
$$

with $c_{1}$ and $c_{2}$ positive real constants. Also, let $\|\Delta(x)\| \leq$ $\delta\|x\|$, where $\delta$ is a nonnegative constant.

If $\delta<\frac{c_{1}}{c_{2}}$, then, the origin is an exponentially stable equilibrium point of the perturbed system (5).

\section{Approximate Input-output Linearization us- ing the Observability Normal Form}

A feature of the input-output feedback linearization is the presence of the internal dynamics $\eta$ in (4). When the input-output system is nonminimum phase, these dynamics are unstable, leading to internal instability. In this paper, the observability normal form is used in conjunction with input-ouput linearization, where a part of the system dynamics is neglected so that the internal dynamics are eliminated upon feedback linearization. 


\subsection{Observability Normal Form for Nonlinear Systems}

Let the vector fields $\left[\frac{\partial h}{\partial x}, \frac{\partial L_{f} h}{\partial x}, \frac{\partial L_{f}^{2} h}{\partial x}, \ldots, \frac{\partial L_{f}^{n-1} h}{\partial x}\right]$ span a space of dimension $n$ for all $x$. This requirement is stronger than the usual notion of observability for nonlinear systems where there is no restriction on the number of Lie derivatives necessary to span the n-dimensional space. Thus, this condition will be referred to as "strong observability". Strong observability implies that the time-varying linearized system is observable around all operating points [14].

For a strongly observable system of relative degree $r$, consider the state transformation $z=\left[h, L_{f} h, L_{f}^{2} h, \ldots, L_{f}^{n-1} h\right]^{T}$ that brings System (1) to the form:

$$
\left[\begin{array}{c}
\dot{z}_{1} \\
\vdots \\
\dot{z}_{r} \\
\vdots \\
\dot{z}_{n}
\end{array}\right]=\left[\begin{array}{c}
z_{2} \\
\vdots \\
z_{r+1} \\
\vdots \\
L_{f}^{n} h
\end{array}\right]+\left[\begin{array}{c}
0 \\
\vdots \\
L_{g} L_{f}^{r-1} h \\
\vdots \\
L_{g} L_{f}^{n-1} h
\end{array}\right] u
$$

Note that $L_{g} L_{f}^{i} h(x)=0, \forall i<r-1$, but $L_{g} L_{f}^{i} h(x) \neq 0$, for $r-1 \leq i<n$. However, if the latter are close to zero, they can be neglected. The approximation $L_{g} L_{f}^{i} h(x)=0$ for $i=r-1, \cdots, n-2$ will be introduced.

Furthermore, if $L_{g} L_{f}^{n-1} h \neq 0$ for all $x$, then the following "linearizing" feedback can be imposed:

$$
u=\frac{v-L_{f}^{n} h}{L_{g} L_{f}^{n-1} h}, \quad v=-K z
$$

with $K=\left[K_{1}, \cdots, K_{n}\right]$. System (9), with the feedback law (10), can be rewritten in the following form:

$$
\dot{z}=A z+\Delta(z)
$$

with:

$$
A=\left[\begin{array}{ccccc}
0 & 1 & 0 & \cdots & 0 \\
0 & 0 & 1 & \cdots & 0 \\
\cdot & \cdots & \cdots & \cdots & \cdot \\
0 & 0 & 0 & \cdots & 1 \\
-K_{1} & -K_{2} & -K_{3} & \cdots & -K_{n}
\end{array}\right]
$$

and:

$$
\Delta(z)=-\left[\begin{array}{c}
0 \\
\cdot \\
\dot{r} \\
L_{g} L_{f}^{r-1} h \\
\dot{ }_{g} L_{f}^{n-2} h \\
0
\end{array}\right] \frac{\left(K z+L_{f}^{n} h\right)}{L_{g} L_{f}^{n-1} h}
$$

\subsection{Stability Analysis}

An interesting observation is that, since $z=0$ is an equilibrium point with $u=0$. Thus, $L_{f}^{n} h(z=0)=0$ from (9). Plugging it in (13) gives $\Delta(0)=0$. So the perturbation is indeed vanishing, and the theory of vanishing perturbations presented in Section 2 can be used.

Theorem 2 Let $\left[\frac{\partial h}{\partial x}, \frac{\partial L_{f} h}{\partial x}, \frac{\partial L_{f}^{2} h}{\partial x}, \ldots, \frac{\partial L_{f}^{n-1} h}{\partial x}\right]$ span a space of dimension $n$ for all $x$. Let $\left|L_{f}^{n} h\right| \leq \delta_{1}\|z\|$ and $\left|L_{g} L_{f}^{n-1} h\right| \geq$ $\delta_{2}>0$. Let $\Delta_{1}$ be defined as:

$$
\Delta_{1}=\left[\begin{array}{c}
0 \\
\cdot \\
L_{g} L_{f}^{r-1} h \\
\cdot \dot{n}-2 \\
L_{g} L_{f}^{n} h \\
0
\end{array}\right]
$$

If

$$
\left\|\Delta_{1}\right\|<\frac{\delta_{2}}{2 \lambda_{\max }(P)\left(\delta_{1}+\|K\|\right)}
$$

where $P$ is the solution of the Lyapunov equation $P A+A^{T} P=$ $-I$, with $I$ the identity matrix and $A$ given by (12), then the feedback law (10) stabilizes (9) exponentially.

Proof: For the nominal system $\dot{z}=A z$, consider the Lyapunov function $V=z^{T} P z$ such that $P$ is a positive symmetric matrix satisfying the Lyapunov equation $P A+A^{T} P=-I$. Then,

$$
\begin{gathered}
\frac{\partial V}{\partial z} A z=-\|z\|^{2} \\
\left\|\frac{\partial V}{\partial z}\right\| \leq 2 \lambda_{\max }(P)\|z\|
\end{gathered}
$$

Considering the same Lyapunov function for the perturbed system (11) leads to:

$$
\begin{aligned}
\dot{V} & =\frac{\partial V}{\partial z} A z+\frac{\partial V}{\partial z} \Delta \\
& \leq-\|z\|^{2}+2 \lambda_{\max }(P)\|z\|\|\Delta\|
\end{aligned}
$$

From (13), and noting that $\left|L_{f}^{n} h\right| \leq \delta_{1}\|z\|$ and $\left|L_{g} L_{f}^{n-1} h\right| \geq$ $\delta_{2}>0$, it follows:

$$
\|\Delta\| \leq \frac{\delta_{1}+\|K\|}{\delta_{2}}\left\|\Delta_{1}\right\|\|z\|
$$

Using (19) in (18) gives

$$
\dot{V} \leq-\|z\|^{2}\left(1-2 \lambda_{\max }(P) \frac{\delta_{1}+\|K\|}{\delta_{2}}\left\|\Delta_{1}\right\|\right)
$$

Thus, if (15) is satisfied, the term in the parenthesis is positive and the feedback law (10) stabilizes (9) exponentially.

The assumption $\left|L_{f}^{n} h\right| \leq \delta_{1}\|z\|$ used in Theorem 2 is a global Lipschitz condition, which is a strong restriction. However, this restriction is necessary to ensure the global stability of the system. If only local Lipschitz conditions are imposed, local stability results could be obtained. 
Corollary 1 For a given gain matrix $K$, the closed-loop system (11) is exponentially stable for all $\left\|\Delta_{1}\right\|<$ $\frac{\delta_{2}}{2 \lambda_{\max }(P)\left(\delta_{1}+\|K\|\right)}$.

The above corollary suggests that, for any given gain matrix $K$, there exists a non-zero range of $\Delta$ for which the system can be stabilized. However, the converse is not true, i.e., given a perturbation $\Delta$, it is not always possible to find a gain matrix $K$ that stabilizes the system.

Corollary 2 Given $\delta_{1}$ and $\delta_{2}$, let

$$
\begin{gathered}
\delta^{*}=\max _{K} \frac{\delta_{2}}{2 \lambda_{\max }(P)\left(\delta_{1}+\|K\|\right)} \\
\text { s.t. } \operatorname{eig}(A)<0
\end{gathered}
$$

For $\left\|\Delta_{1}\right\|<\delta^{*}$, there exists a $K$ that exponentially stabilizes System (11).

The above maximization can be done numerically for given values of $\delta_{1}$ and $\delta_{2}$.

\section{Simulation Example}

The following nonlinear system is considered to illustrate the theory presented above:

$$
\begin{aligned}
\dot{x}_{1} & =x_{2} \\
\dot{x}_{2} & =x_{3}-\alpha u \\
\dot{x}_{3} & =x_{1}+\left(1+x_{1}^{2}\right) u \\
y & =x_{1}
\end{aligned}
$$

with $\alpha$ being a positive constant. The vector fields $f, g$, and $h$ are given by:

$$
\begin{gathered}
f(x)=\left[\begin{array}{l}
x_{2} \\
x_{3} \\
x_{1}
\end{array}\right], \quad g(x)=\left[\begin{array}{c}
0 \\
-\alpha \\
1+x_{1}^{2}
\end{array}\right] \\
h(x)=x_{1}
\end{gathered}
$$

For System (22), the Lie brackets $[f, g]$ and $[g,[f, g]]$ are:

$$
[f, g]=\left[\begin{array}{c}
\alpha \\
-1-x_{1}^{2} \\
2 x_{1} x_{2}
\end{array}\right], \quad[g,[f, g]]=\left[\begin{array}{c}
0 \\
0 \\
-4 \alpha x_{1}
\end{array}\right]
$$

Since $\operatorname{det}([g,[f, g],[g,[f, g]]])=-4 \alpha^{3} x_{1}$, the distribution $\operatorname{span}\{g,[f, g]\}$ is not involutive. Thus, System (22) is not fullstate feedback linearizable [8]. Note that for $\alpha=0$, the system (22) would be full-state feedback linearizable.

Note that the present example has been carefully constructed, so as to illustrate the proposed approximate input-output linearization. The assumptions of Theorem 2 are automatically verified, and System (22) is already written in the observability canonical form.

In the following subsections, three schemes are compared: (i) standard input-output feedback linearization with $y=x_{1}$, (ii) input-output feedback linearization with a minimum-phase output $y_{m p}=x_{1}+x_{2}+x_{3}$, and (iii) approximate input-output linearization with $y=x_{1}$.

The simulation results are for $\alpha=0.03$ and the initial state $x_{0}=[1,1,1]^{T}$. The gains for the various schemes are adjusted so that the input amplitudes are comparable in size.

\subsection{Standard Input-output Linearization}

Since the first Lie derivatives of the output are $L_{g} h=0$ and $L_{g} L_{f} h=-\alpha \neq 0$, System (22) has relative degree two. The input-output linearization technique that imposes $v=-\tilde{K}_{1} x_{1}-\tilde{K}_{2} x_{2}, \tilde{K}_{1}, \tilde{K}_{2}>0$ leads to the linear controller:

$$
u=\frac{v-L_{f}^{2} h}{L_{g} L_{f} h}=\frac{\tilde{K}_{1} x_{1}+\tilde{K}_{2} x_{2}+x_{3}}{\alpha}
$$

However, at the equilibrium, $y=x_{1}=0, \dot{y}=x_{2}=0$. The input is then $u=\frac{x_{3}}{\alpha}$, which gives the zero dynamics: $\dot{x}_{3}=\frac{x_{3}}{\alpha}$. Since $\alpha$ is positive, the zero dynamics are unstable. Also, the zero location is at $\frac{1}{\alpha}$. Therefore, System (22) is nonminimum phase. The evolution of the system for $\tilde{K}_{1}=100$ and $\tilde{K}_{2}=20$ is shown in Figure 1. It can be seen that the internal state $x_{3}$ and the input $u$ escape to infinity.
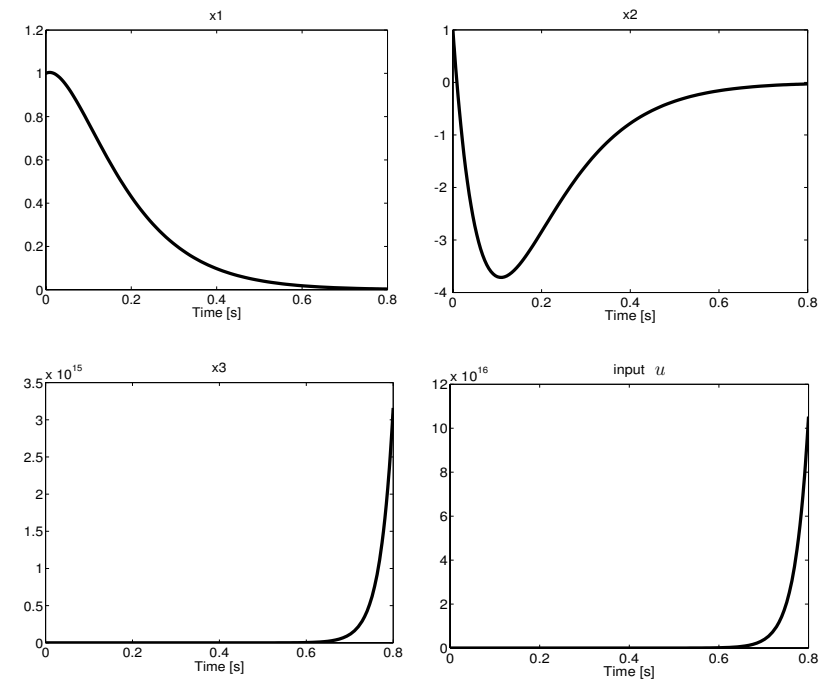

Figure 1: Evolution of the states and input of the controlled system using standard input-output linearization

\subsection{Input-output Linearization with Minimum-phase Output}

One possibility to avoid instability is to find an output for which the system is minimum phase so that standard input-output linearization leads to stable internal dynamics. For this simple system, it is easy to obtain such an output: $y_{m p}=h_{m p}(x)=$ 
$x_{1}+x_{2}+x_{3}$.

The Lie derivatives of the nonminimum-phase output are $L_{g} h_{m p}=1-\alpha+x_{1}^{2}, L_{f} h_{m p}=x_{1}+x_{2}+x_{3}$. Thus, if $\alpha<1$, the relative degree is one for all $x$. Imposing $v=-\bar{K} y_{m p}$, $\bar{K}>0$, the controller becomes:

$$
u=\frac{v-L_{f} h_{m p}}{L_{g} h_{m p}}=\frac{-(\bar{K}+1)\left(x_{1}+x_{2}+x_{3}\right)}{1-\alpha+x_{1}^{2}}
$$

At equilibrium $y_{m p}=x_{1}+x_{2}+x_{3}=0$. Thus, the input is equal to $u=0$. Substituting $x_{3}=-x_{1}-x_{2}$ gives the stable zero dynamics $\dot{x}_{1}=x_{2}, \dot{x}_{1}=-x_{1}-x_{2}$, thereby confirming that the chosen output is indeed minimum phase.

Simulation results are shown in Figure 2 for $\bar{K}=0.44$. The minimum-phase output converges towards its desired equilibrium value, while the states follow it with a time lag.
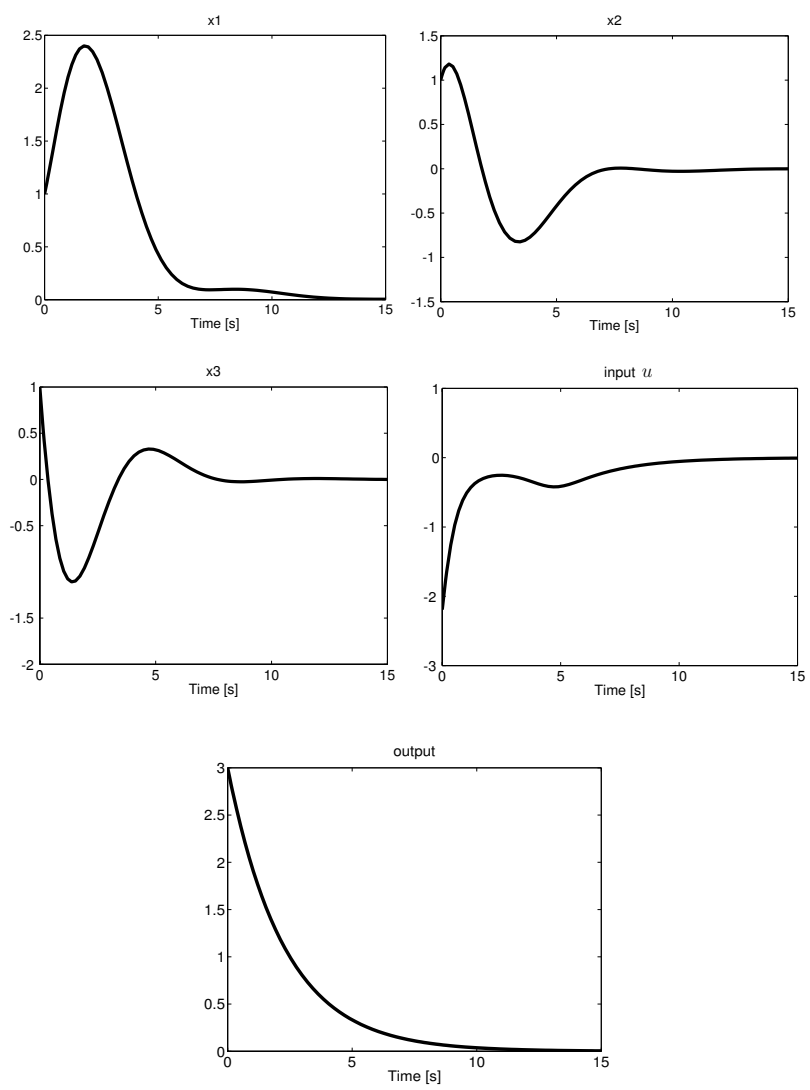

Figure 2: Evolution of the states, output and input of the controlled system using input-output linearization with minimumphase output

\subsection{Approximate Input-output Linearization Using the Observability Normal Form}

The proposed approximate input-output linearization is applied to System (22). The Lie derivatives are computed up to the order of the system: $h=x_{1}, L_{f} h=x_{2}, L_{f}^{2} h=x_{3}, L_{g} h=0$, $L_{g} L_{f} h=-\alpha$, and $L_{g} L_{f}^{2} h=1+x_{1}^{2}$. Since $h, L_{f} h$ and $L_{f}^{2} h$ are independent, the system can be written in the normal form
(9). In fact, System (22) is already in that normal form.

Imposing the linear controller, $v=-K_{1} x_{1}-K_{2} x_{2}-K_{3} x_{3}$ gives:

$$
u=\frac{v-L_{f}^{3} h}{L_{g} L_{f}^{2} h}=\frac{-\left(K_{1}+1\right) x_{1}-K_{2} x_{2}-K_{3} x_{3}}{1+x_{1}^{2}}
$$

Applying Theorem 2 to prove stability, with $\Delta_{1}=[0-\alpha 0]^{T}$, the condition on $\alpha$ becomes:

$$
\alpha<\frac{1}{2 \lambda_{\max }(P)(1+\|K\|)}
$$

The positive matrix $P$ is solution of the Lyapunov equation $P A+A^{T} P=-I$, where $I$ is the identity matrix of dimension 3 and $A$ is given by:

$$
A=\left[\begin{array}{ccc}
0 & 1 & 0 \\
0 & 0 & 1 \\
-K_{1} & -K_{2} & -K_{3}
\end{array}\right]
$$

The gain values that maximize the right-hand side of inequality (26) are $K_{1}=0.7981, K_{2}=2.2103$ and $K_{3}=1.3668$, leading to $\alpha_{\max }=0.03$. However, the vanishing perturbation theory is very conservative: $\alpha_{\max }$ indicated by the theory is much smaller than the value that can actually be accommodated. Trial and error simulations have indicated that, with the same controller, it is possible to stabilize the system up to $\alpha=0.6$. Simulation results using the control law (25) are presented in Figure 3. It can be seen that the system is asymptotically stable.
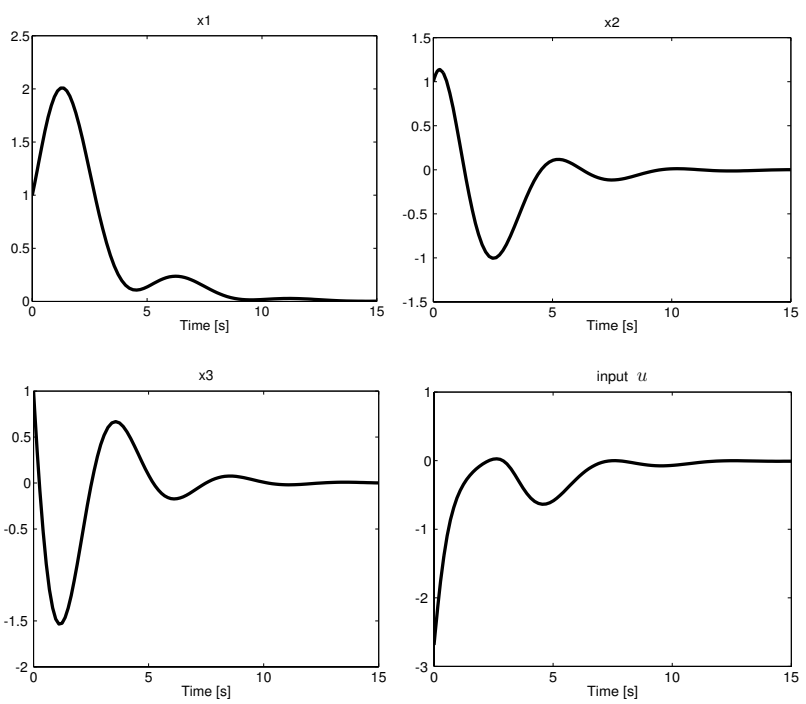

Figure 3: Evolution of the states and input of the controlled system using approximate input-output linearization using the observability normal form

Comparing Figures 2 and 3 leads to the interesting observation that the inputs in the last two schemes are fairly close and lead to similar results, despite the fact that they are calculated using 
different outputs. This stems from the fact that the control laws (24) and (25) are fairly close numerically:

$$
\begin{aligned}
& u_{1}=\frac{-1.44 x_{1}-1.44 x_{2}-1.44 x_{3}}{0.97+x_{1}^{2}} \\
& u_{2}=\frac{-1.79 x_{1}-2.21 x_{2}-1.36 x_{3}}{1+x_{1}^{2}}
\end{aligned}
$$

where $u_{1}$ is the control law resulting from the input-output feedback linearization with minimum-phase output, and $u_{2}$ is the control law resulting from the proposed approximate inputoutput linearization.

\section{Conclusion}

This paper has presented a control scheme based on an approximate input-output linearization using the observability normal form. The transformed system is approximated as a chain of integrators, and the neglected part is considered as a perturbation. Since the perturbation is a vanishing one, stability analysis based on vanishing perturbation theory is also provided.

Unfortunately, the stability results presented here can be very conservative. In the example considered, a much larger perturbation could have been accommodated than that predicted by the theoretical results. It may be possible to enlarge the domain of stability by including the perturbation into the control law instead of just neglecting it. This will be investigated in future work.

The stability or instability of the internal dynamics are related to the location of the zeros of the linearized original dynamics. By eliminating the internal dynamics, all the zero locations are pushed to infinity (no zeros). So, it can be postulated that the faster the zeros, the more valid the approximation, as was confirmed by the simulation example. However, a more formal link between the zero locations and the norm of the vanishing perturbation requires additional investigation.

\section{References}

[1] F. Allgöwer. Approximate input-output linearization of nonminimum phase nonlinear systems. In European Control Conference, page 604, Brussels, Belgium, 1997.

[2] S. A. Bortoff. Approximate state-feedback linearization using spline functions. Automatica, 33(8):1449-1458, 1997.

[3] D. Chen and B. Paden. Stable inversion of nonlinear nonminimum phase systems. International Journal of Control, 64(1):81-97, 1996.

[4] S. Gopalswamy and J. Hedrick. Tracking nonlinear nonminimum phase systems using sliding control. International Journal of Control, 57(5):1141-1158, 1993.

[5] G. O. Guardabassi and S. M. Savaresi. Approximate linearization via feedback - an overview. Automatica, 37:115,2001 .
[6] K. Guemghar, B. Srinivasan, P. Mullhaupt, and D. Bonvin. Predictive control of fast unstable and nonminimumphase nonlinear systems. In American Control Conference, pages 4764-4769, Anchorage, USA, 2002.

[7] R. Gurumoorthy and S. R. Sandres. Controlling nonminimum phase nonlinear systems - the inverted pendulum on a cart example. In American Control Conference, pages 680-685, Evanston,USA, 1993.

[8] A. Isidori. Nonlinear Control Systems. Springer-Verlag, 1989.

[9] J. M. Kanter, M. Soroush, and W. D. Seider. Continuoustime, nonlinear feedback control of stable processes. Ind. Eng. Chem. Res., 40:2069-2078, 2001.

[10] H. K. Khalil. Nonlinear Systems. Prentice Hall, New Jersey, 1996.

[11] M. Krstic, I. Kanellakopoulos, and P. Kokotovic. Nonlinear and Adaptive Control Design. Wiley and sons, New York, 1995.

[12] D. A. Laurence and W. J. Rugh. Input-output pseudolinearization for nonlinear systems. IEEE Transactions on Automatic Control, 39(11):2207-2218, 1994.

[13] M. Niemiec and C. Kravaris. Nonlinear model-state feedback control for nonminimum-phase processes. Automatica, 39(7):1294-1302, 2003.

[14] H. Nijmeijer and A. J. Van Der Schaft. Nonlinear Dynamical Control Systems. Springer-Verlag, 1990.

[15] R. Ortega, A. J. Van der Schaft, I. Mareels, and B. Maschke. Energy shaping revisited. In IEEE International Conference on Control Applications, pages 121125, Anchorage, USA, 2000.

[16] C. Reboulet and C. Champetier. A new method for linearizing nonlinear systems: The pseudolinearization. International Journal of Control, 40:631-638, 1984.

[17] M. Soroush and C. Kravaris. A continuous-time formulation of nonlinear model predictive control. International Journal of Control, 63(1):121-146, 1996.

[18] M. W. Spong and M. Vidyasagar. Robot Dynamics and Control. Wiley and sons, New York, 1989.

[19] J. Wang and W. J. Rugh. On the pseudo-linearization problem for nonlinear systems. Systems and Control Letters, 12:161-167, 1989. 